\title{
Many Worlds: Decoherent or Incoherent?
}

\author{
Richard Dawid $^{* 1}$ and Karim Thébault ${ }^{\dagger 2}$ \\ ${ }^{1}$ Institut für Philosophie, Universität Wien, Universitätsstraße 7, A-1010 Vienna, Austria \\ ${ }^{2}$ MCMP, Ludwig Maximilians Universität, Ludwigstrasse 31, D-80539, Munich, Germany
}

January 23, 2013

\begin{abstract}
The Deutsch-Wallace-Everett programme is conceptually incoherent since its viability rests upon a notion of decoherence that conflicts with its own fundamental precepts in two respects. These problems are not alleviated by invoking the notion of emergent or robust structure, and are argued to be significant enough to cast doubt upon the viability of the entire neo-Everettian enterprise as it now stands.
\end{abstract}

*richard.dawid@univie.ac.at

${ }^{\dagger}$ karim.thebault@gmail.com 


\section{Contents}

1 Introduction $\quad 2$

2 The Deutsch-Wallace-Everett approach to Quantum Mechanics 3

2.1 Probability and decoherence . . . . . . . . . . . . . . . . . . . 3

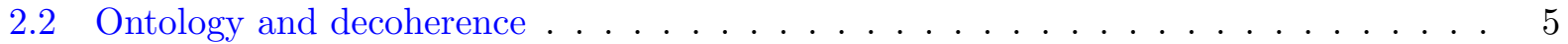

3 Decoherence, circularity and incoherence: objecting to the Deutch-Wallace$\begin{array}{ll}\text { Everett approach to quantum mechanics } & 6\end{array}$

3.1 Circularity and the Zurek contention . . . . . . . . . . . . . . . . . . 6

3.2 Not just circular but incoherent . . . . . . . . . . . . . . . . . . . . . 9

3.3 Incoherence and ontological prejudice . . . . . . . . . . . . . . . . 12

4 Emergent worlds? 14

$\begin{array}{llr}5 & \text { Conclusion } & 17\end{array}$

\section{Introduction}

In recent years, Everett-type approaches have assumed an eminent, if not quite pre-eminent, position among the various interpretations of quantum mechanics. This rise in the perceived persuasiveness of Everettian arguments can be related to two significant new developments. First, it was understood that decoherence could provide a foundation for an interpretation of the wave function in terms of a superposition of branches which, after forking off, remained effectively independent from each other. Second, it was suggested that a satisfactory Everettian interpretation of quantum mechanics could be based on a purely subjective ${ }^{1}$ perspective on the probabilistic role of the wave function. According to this understanding, the connection between the wave function and probabilities does not rely on any objective characteristics of the many worlds scenario (be it objective probabilities, numbers of branches or other) but is rather a product of the rational behaviour of agents who find themselves in one branch of the Everettian universe.

This new, subjectivist understanding of Everettian quantum mechanics was given a formal basis through the work of Deutsch [1] and Wallace [2, 3, 4] (see also [5]); with their proofs demonstrating that, provided Everettian quantum mechanics is true, the Born rule can be established, under certain conditions, as being constitutive of any rational betting behaviour on future outcomes of quantum experiments.

Various critical responses to this Deutsch-Wallace-Everett (DWE) approach to quantum theory have been put forward. A majority of these focus upon the the structure of the decision theoretic proof itself, and rely either on disputing the notion of rationality [6], or on challenging the derivation

\footnotetext{
${ }^{1}$ Here the use of 'epistemic' rather than 'subjective' would perhaps be more consistent with the terminology in use within the philosophy of science. However, we will retain the original terminology to avoid confusion.
} 
of the Born rule as a unique/viable subjective probabilistic measure [7, 8]. Such internal criticisms rest upon implicitly accepting the viability of the DWE framework but questioning particular aspects of its implementation. Another important line of criticism seeks to undermine the DWE claim of a derivation of the Born rule on the basis of a decoherence related circularity objection $[9,10,11,12]$. A further perceived problem relates to the role played by probabilistic empirical phenomena within the the DWE approach. Prima facie, within the DWE framework as it stands there seems no natural connection between the part played the Born weighting, as assigned to branches, and empirical statistical data that can be observed within a single world situation, and this might be seen to cast doubt upon the empirical viability of the approach. ${ }^{2}$

Thus, various authors have already found reasons to claim that the DWE approach has certain, major conceptual flaws. In the present paper, we want to make a stronger claim. We will argue that the DWE programme is conceptually incoherent since its viability rests upon a notion of decoherence that conflicts with its own fundamental precepts.

This problem is argued to be a severe one and is, as we shall see in Section 4, not alleviated by invoking the notion of emergent or robust structure. Properly considered, we believe the incoherence issues identified are significant enough to cast doubt upon the entire neo-Everettian enterprise as it now stands.

\section{The Deutsch-Wallace-Everett approach to Quantum Mechanics}

\subsection{Probability and decoherence}

Assume that an agent carries out a quantum mechanical experiment with a number of different possible outcomes. Broadly speaking, Everett-type interpretations of quantum mechanics relate each one of the possible distinct outcomes to a particular branch within a 'many worlds' universe. We understand this universe as including a representation of every physically possible outcome of this and every other experiment (where, under some understandings, 'experiments' are taken to include a variety of spontaneous physical processes); and we understand these representations to exist within an ordered branching tree structure, where a set of worlds following on from every distinct outcome of every experiment can be identified with its own independent 'sub-tree'. If we assume that our experiment is the initial branching event (which is equivalent to focusing in on one particular sub-tree), then immediately subsequently we can think of a many worlds universe as constituted purely by an array of worlds, with one world corresponding to each possible outcome of our experiment.

Now, since an instantiation of our agent exists in each one of these worlds, the agent's chances of finding themselves in one specific world after the experiment might be expected to be directly determined by the proportion of worlds within which the relevant experimental outcome obtained. In the tree picture, this is of course just equivalent to counting the number of branches with the relevant outcome that fork off from the initial state of the experiment, and then dividing this by the

\footnotetext{
${ }^{2}$ See [13] for further discussion of this and related issues.
} 
total number of branches. Intuitively then, one may expect that within an Everettian picture the probabilities relevant to the outcomes of quantum mechanical experiments can be obtained simply via such a branch counting methodology. However, it transpires that if we consider an agent who adopts a branching counting strategy, they will not, in general, reproduce the probabilities provided by quantum mechanics (see e.g. [14]). Thus, to make sense of probability in an Everettian setting we require both: i) a good reason to discount the viability of the (seemingly intuitive) branch counting strategy; and ii) an alternative methodology leading to the correct Born rule measure.

According to the Deutsch-Wallace argument (on this see in particular [3, §9]) it is decoherence effects - which, as discussed below, are in any case needed to ground the discreetness of the branches - that provide the basis for an argument towards the impossibility of extracting probability statements on the outcomes of quantum experiments from branch counting. Since decoherence is fuzzy in the sense that there is no precise way of telling at what point in time it occurs, it is impossible to specify one definitive branching structure for a quantum process. This in turn implies that no definitive probabilistic conclusions can be drawn from branch counting since the number of branches is inherently indeterminate. We should thus conclude that quantum probabilities cannot be implemented at an objective level in an Everettian framework at all. Once the connection between measurement probabilities and the branching structure is broken the problem of an incompatibility between the two concepts disappears.

It still remains to be seen, however, exactly how measurement probabilities are related to Everettian QM at all. The second step in the Deutsch-Wallace argument is to implement probabilities at a subjective level by introducing a decision theoretic analysis from the perspective of an agent within the branching structure. Immediately before a branching event it is rational, taking the perspective of an embedded agent, for me to consider the interests of my future selves in the various branches. Even though there is no fact of the matter about which future self $I$ will become, I should believe that I will become exactly one of them and, given it is uncertain which one I will become, my current deliberations (in particular betting behaviour) should then be dictated by the combination of my preference between the various possible eventualities and the extent to which I think they are likely to be relevant to me.

The key to the Deutsch-Wallace argument is then to demand that the ordering an agent in such a position of subjective uncertainty ${ }^{3}$ can apply to their preferences between the various outcomes, is constrained by a set of basic rationality criteria (with the specifics differing slightly between the various proofs). These rationality criteria are taken to be necessary for consistent reasoning and include both seemingly self-evident conditions, such as coherence, and several more disputable principles, such as diachronic consistency. Given this basis, the principal achievement of DeutschWallace proofs is then the demonstration that, up to linear transformations, we can fix the Born rule as the only consistent subjective probability measure available to our deliberating agent.

\footnotetext{
${ }^{3}$ See [15] for detailed philosophical consideration of this idea and [16, §6.10.4] for consideration of its changing role within Wallace's arguments. An alternative 'objective determinism' understanding of personal identity in this context has also been considered, see [3] and references therein for details. This distinction is not important for the purposes of the arguments given here.
} 
As noted above, questions have been raised with regard to both the cogency of the rationality criteria chosen, and the uniqueness of the relevant proofs. We will not here add to the already substantial literature in this line. Rather, we will, for the sake of argument, accept that once the first step of breaking the connection between objective branching structure and probabilities via decoherence arguments has been achieved, a derivation of the Born rule as a subjective probability measure along decision theoretic lines can be established. Thus, from our perspective, the crucial issues with regard to probability in the DWE framework, will turn on whether or not decoherence effects can consistently support the initial argumentative move to dismiss objective quantum mechanical probability measures altogether.

\subsection{Ontology and decoherence}

The central Everettian claim, dating back to the work of DeWitt and Everett himself, is that that theirs is the interpretation which is naturally implied by the bare mathematical formalism of quantum theory - in particular, the unitary evolution of the quantum state is taken to be a complete description of quantum dynamics. As we have seen, the Everettian ontology is constituted by some form of branching tree structure with each branch corresponding to a discrete outcome of events. Quantum mechanically the instantaneous state of such a branching structure corresponds to a proper mixture of pure states: the branches can be understood as decoupled from each other. It is from the perspective of an agent situated within this ontology (or something that closely approximates it) that the Deutsch-Wallace argument, based upon classical decision theory, is applied.

Within the quantum formalism there can, of course, exist states which cannot be understood in terms of proper mixtures of pure states. When considering quantum systems we can, and generically do, encounter quantum states which are irreducibly superpositions of different pure states and thus correspond to non-classical interference between distinct outcomes. Such superposition states cannot be represented in terms of a discrete branching tree structure and are inherently at conflict with any application of classical decision theory. In other words, the observation that we do not see macroscopic entanglement is a precondition for any notion of a classical rational agent existing within a many worlds framework. Thus, we can insist that proponents of Everettian quantum theory in general, and those who apply classical decision theory within it in particular, must provide a mechanism for removing or discounting superposition states in the context of macroscopic phenomena at the very least. In order to remain consistent with their own precepts they must do this in the context of a formalism where the fundamental dynamics is unitary.

The move that is supposed to address this tension is the invocation of decoherence: a quasiclassical branching structure can be shown to emerge from a fundamentally quantum mechanical underlying reality based on the unitary evolution of the system. Thus, according to Wallace [17], the Everettian can claim both that the quantum state is all there is and that there exist 'worlds' which are mutually isolated structures instantiated within the dynamical state. We can thus understand decoherence as a 'dynamical process by which two components of a complex entity (the quantum 
state) come to evolve independently of one another, and it occurs due to rather high-level, emergent consequences of the particular dynamics and initial state of our Universe.' [17, p.10]

Crucially, this means that rather than eliminating the superposition states associated with quantum mechanical interference, we are supposed to think of decoherence processes as merely rendering their contribution to the quantum state of complex systems negligible for all practical purposes. Thus, the discrete branching ontology fundamental to the many worlds picture in general, and the Deutsch-Wallace subjective probability approach in particular, is not claimed to be established by a direct decoherence algorithm starting with the quantum state. Rather, it is claimed, the ubiquity of decoherence phenomena for reasonably complex systems on reasonably long time scales means that one may justifiably treat the quantum mechanical description of high-level systems as effectively describing a many worlds ontology.

\section{Decoherence, circularity and incoherence: objecting to the Deutsch- Wallace-Everett approach to quantum mechanics}

It is crucial to the coherence of the DWE framework that by by invoking 'decoherence theory' the Everettian is not introducing formal or conceptual machinery to which they are not entitled. In particular, no notion of probability or ontology that is in direct conflict with the relevant Everettian notions can consistently be relied upon to argue towards those notions, otherwise the scheme would be conceptually incoherent. Furthermore, given the proponents of the DWE scheme claim that the decision theoretic proofs enable them to derive the Born rule within the post-decoherence many worlds structure, the non-circularity of such a derivation depends on the possibility of invoking decoherence effects without the need to assume the Born rule. In this section we will investigate three objections - two of incoherence one of circularity - all based upon a close analysis of the details of decoherence.

\subsection{Circularity and the Zurek contention}

Let us follow the account given by Zurek [18] and consider the case of a simple set-up consisting of a two state, spin-half system $\mathcal{S}$ and a quantum detector $\mathcal{D}$. If we represent the two states of the detector as $|\uparrow\rangle$ and $|\downarrow\rangle$ then we can simply consider a detector (also with two states) which is such that:

$$
\begin{aligned}
& |\uparrow\rangle\left|d_{\downarrow}\right\rangle \rightarrow|\uparrow\rangle\left|d_{\uparrow}\right\rangle \\
& |\downarrow\rangle\left|d_{\downarrow}\right\rangle \rightarrow|\downarrow\rangle\left|d_{\downarrow}\right\rangle
\end{aligned}
$$

Before the interaction between system and detector we can represent the initial state of the composite system as:

$$
\left|\Phi^{I}\right\rangle=(\alpha|\uparrow\rangle+\beta|\downarrow\rangle)\left|d_{\downarrow}\right\rangle
$$


and after the interaction the quantum state of the composite system is:

$$
\left|\Phi^{C}\right\rangle=\alpha|\uparrow\rangle\left|d_{\uparrow}\right\rangle+\beta|\downarrow\rangle\left|d_{\downarrow}\right\rangle
$$

The process that connects these two states is simply evolution according the the Schrodinger equation; such states are, therefore, given by the quantum formalism on its own. That this quantum state can not be understood as a proper mixture of distinct outcomes can be seen easily by considering the relevant density matrix:

$$
\begin{array}{r}
\rho^{C}=|\alpha|^{2}|\uparrow\rangle\left\langle\uparrow|| d_{\uparrow}\right\rangle\left\langle d_{\uparrow}\left|+\alpha \beta^{*}\right| \uparrow\right\rangle\left\langle\downarrow|| d_{\uparrow}\right\rangle\left\langle d_{\downarrow}\right| \\
\quad+\alpha^{*} \beta|\downarrow\rangle\left\langle\uparrow|| d_{\downarrow}\right\rangle\left\langle\left. d_{\uparrow}|+| \beta\right|^{2} \mid \downarrow\right\rangle\left\langle\downarrow|| d_{\downarrow}\right\rangle\left\langle d_{\downarrow}\right|
\end{array}
$$

The off diagonal elements of this matrix if taken ontologically seriously would correspond to purely quantum phenomena where outcomes are superposition states rather than distinct pure states. Compare this to a reduced destiny matrix without the off diagonal elements:

$$
\rho^{R}=|\alpha|^{2}|\uparrow\rangle\left\langle\uparrow|| d_{\uparrow}\right\rangle\left\langle\left. d_{\uparrow}|+| \beta\right|^{2} \mid \downarrow\right\rangle\left\langle\downarrow|| d_{\downarrow}\right\rangle\left\langle d_{\downarrow}\right|
$$

This we can interpret as a proper mixture of pure states and so taking it ontologically seriously would seem to imply the discrete branching structure which the Everettian requires. The idea is then that we can understand decoherence as a process by which $\rho^{C} \Rightarrow \rho^{R}$ through the (unitary) interaction of the composite system and the environment. One assumes an interaction between system and environment of the form:

$$
\left.\left.\left|\Phi^{C}\right\rangle\left|\mathcal{E}_{C}\right\rangle=\alpha|\uparrow\rangle\left|d_{\uparrow}\right\rangle \mathcal{E}_{\uparrow}\right\rangle+\beta|\downarrow\rangle\left|d_{\downarrow}\right\rangle \mathcal{E}_{\downarrow}\right\rangle=|\Psi\rangle
$$

The claim is that when the states of the environment corresponding to the detector states are orthogonal - i.e. we have that $\left\langle\mathcal{E}_{i} \mid \mathcal{E}_{i^{\prime}}\right\rangle=\delta_{i i^{\prime}}$ - the density matrix for the original detector-system combination post-decoherence is given by the expression:

$$
\rho^{\mathrm{PD}}=\operatorname{Tr}_{\mathcal{E}}|\Psi\rangle\left\langle\left.\Psi\left|\Sigma_{i}\left\langle\mathcal{E}_{i} \mid \Psi\right\rangle\left\langle\Psi \mid \mathcal{E}_{i^{\prime}}\right\rangle \cong\right| \alpha\right|^{2} \mid \uparrow\right\rangle\left\langle\uparrow|| d_{\uparrow}\right\rangle\left\langle\left. d_{\uparrow}|+| \beta\right|^{2} \mid \downarrow\right\rangle\left\langle\downarrow|| d_{\downarrow}\right\rangle\left\langle d_{\downarrow}\right|=\rho^{R}
$$

Environment induced decoherence does not fully eliminate the off diagonal elements, but it re-scales them to vanishingly small amplitudes as given by the associated Born weights (see the explicit formulas of [18] as well as references therein). In order for environment induced decoherence to give us licence to ignore the superposition states - and therefore justify an interpretation of the quantum state in terms of a discrete branching - we need one more step: we have to find a reason for neglecting the off-diagonal terms due to their small values.

This can only be done, however, once we have an understanding as to what the numbers we write into the density matrix mean. The simple statement that after evolving a quantum system 
relative to a background environment we can consistently attribute small numbers to entangled states, has no relevance as long as we do not know what those numbers stand for. Obviously, we cannot always neglect small parameter values. Neither would it make sense to neglect the early stages of the universe on the grounds that the values of the time parameter are small, nor to consider the electroweak interaction as relevant only when the value of the distance parameter is high. In order to neglect small values in favour of larger values, we have to establish that the magnitude of the corresponding variable is related to the entry's effect on the measurement to be performed. Since experimental testing and the entries in the density matrix are related in terms of the probabilities for measuring certain outcomes, in order to establish the negligibility of small entries in the density matrix we must introduce the Born rule. Following the influential analysis of Zurek [9, p.25], we can state this crucial contention simply as:

Z Decoherence cannot be practiced without an independent prior derivation (or assumption) of the Born rule.

A significant consequence of $\mathbf{Z}$ for the Deutsch-Wallace type derivation of the Born rule in terms of subjective probability has been emphasised first by Zurek himself [9, p.25], and then by Baker [11], and Kent [12]. In essence all three authors argue that, since decoherence must be assumed in order to ground the DWE classical decision theoretic framework, and since by invoking decoherence one is inevitably assuming the Born rule, the derivation of the Born rule within the DWE scheme is inherently circular. Kent summaries this circularity objection as follows:

Even if one could show, as Wallace claims, that agents defined within that ontology are rationally justified in using the Born rule as a calculus for decisions, it would seem incorrect to portray this argument as a derivation of the Born rule within Everettian quantum theory. Wallace's argument should rather be understood as attempting to show something weaker: that the Born rule re-emerges as output (albeit, to be fair, in an interesting and non-obvious way) if assumed as input. [12, p.17]

Similarly, Baker specifically builds his detailed presentation of the objection around the need to assume decoherence before we can consider the perspective of an agent making decisions with regard to the distinct outcomes of quantum process (i.e. an agent playing a 'quantum game'):

I claim that, since the employment of decoherence to identify branches depends upon the unlikeliness of low-weight events, the framework of quantum games in which the theorem is formulated presumes its conclusion. Unless the Born rule (or some similar rule permitting inferences from low weight to low probability) holds, there are no quantum games in Everett. [11, p.21]

We thus see that the Zurek-Baker-Kent circularity objection to the DWE programme follows in straightforward terms from the combination of the Zurek contention, $\mathbf{Z}$ above, together with 
the fairly self-evidently necessary requirement for proponents of the DWE scheme to assume decoherence prior to launching into decision theoretic calculus that eventually leads to the relevant probability measure: Without decoherence there is no classical agential perspective, and without this there is no basis for a Deutsch-Wallace type derivation of the Born rule. We can formalise the situation as follows:

- Z. Decoherence cannot be practiced without an independent prior derivation (or assumption) of the Born rule.

- P1. Decoherence effects are a necessary precondition for one to assume the perspective of an agent making decisions (i.e. playing quantum games) within a world of distinct outcomes, corresponding to the discrete branches of the many worlds ontology.

- P2. Adopting this agential perspective is necessary prior to any Deutch-Wallace type derivation of the Born rule as a subjective probability measure.

Therefore:

- C. Deutsch-Wallace type derivations of the Born rule as a subjective probability measure are inherently circular since they involve the prior assumption of the Born rule in order to establish the Born rule

Thus there exists a seemingly robust challenge to any Everttian claim of being able to derive the Born rule based upon arguments of the Deutch-Walalce type. For all that, even if the Everettians are forced to accept that they cannot, as claimed, derive the Born rule ${ }^{4}$ the Deutsch-Wallace argument might seem to at least suggest consistency within the framework, and to possibly also give an insight into the much discussed connection between objective and subjective notions of probability [20]. However, as claimed above, the conceptual problems posed by decoherence for the neo-Everettian approach go beyond an objection merely on the grounds of circularity.

\subsection{Not just circular but incoherent}

In the analysis above we saw that decoherence processes do not provide the foundations necessary for establishing the DWE scheme without the prior assumption of a notion of probability as given by the Born rule. This is because although such processes may act to re-scale the weightings of the superposition states to be very small, the interpretation of the smallness of the parameter as indicating the neglectability of the relevant entry in the density matrix relies on the prior assumption of the Born rule. Implicit within this part of our argument towards $\mathbf{Z}$ is that when we are considering the Born rule within a decoherence context, it is as a rule for relating the quantum

\footnotetext{
${ }^{4}$ They would, in this respect, then be at the disadvantage of their Bohmian rivals who's own derivation would not seem to be susceptible to the same objections on the grounds of circularity [19].
} 
formalism to some objective empirical feature of the ontology in question. ${ }^{5}$ In other words, in assuming the Born rule for decoherence we must assume either that the squared absolute value of the wave function corresponds to the objective probability that the corresponding experimental outcome is in fact realised or that it corresponds to some other empirical structure from which an objective probabilistic statement can be derived. Only once this is achieved can we have a basis for neglecting any entries in the density matrix. We can thus be a little more specific than above and argue that the decoherence process which the Everettian employs will inevitably involve some prior notion of objective probabilities given by the Born rule, otherwise the required quasiclassical branching structure ontology cannot be established. The probabilities corresponding to the properties on which decoherence relies must be objective since decoherence is used to induce the individuation of branches, which in turn is a necessary precondition for establishing a subjective agent's perspective at all. We clearly cannot, therefore, meaningfully define a subjective notion of probability until the stage after deploying decoherence. ${ }^{6}$ This allows us to reformulate the Zurek contention as follows:

Z* Decoherence cannot be practiced without an independent prior derivation (or assumption) of the Born rule as an objective probability measure.

Recall from $\S 2.1$ that we saw it was necessary for the proponent of the DWE scheme to argue that quantum probabilities cannot be implemented at an objective level in an Everettian framework because of the need to avoid the inconsistency between (objective) probabilities given by a branch counting strategy, and those corresponding to Born weightings. In more general terms, we can argue that the viability of the DWE scheme rests upon there being no possible objective implementation of probability in the Everettian framework. In order to see this even more clearly, let us assume for a moment that predictions regarding the statistics of quantum processes could be extracted at the objective level. In that case, one could distinguish two possible outcomes: either those predictions would or they would not be in agreement with the Born rule. In the first case, one could use that result immediately in support of Everettian QM and the deployment of the decision theoretic arguments would be entirely superfluous. We would adopt an objective rather than a subjective Everettian position since such a position would be directly supported by the formalism. In the second case, we would have a direct contradiction between Everettian quantum mechanics and observation. No decision theoretic argument could remove that contradiction and Everettian QM would be considered refuted by empirical data. The claim that a decision theoretic argument can

\footnotetext{
${ }^{5}$ We will for the time being assume only one ontological regime, and therefore neglect the the possible implications of viewing the many worlds ontology as emergent. A detailed consideration of whether a line of argument based upon an emergent notion of ontology allow the proponent of the DWE scheme to rebut our incoherence objections will be made in the following section.

${ }^{6}$ This suggests a general principle defining a limit to the explanatory role of subjective probability with regard to the probabilistic structure of a physical theory. The primary precept: a subjective notion of probability cannot be used to account for objective probabilistic features of a theory that are themselves necessary to invoke the perspective of agents within the theory. This is (in a sense) the complement to Lewis' [21] principal principle that (in the terminology we are using) subjective probability should be constrained by objective probability.
} 
be used for establishing the viability of Everettian QM mechanics thus must rely on the assumption that no probabilistic implications of Everettian QM can be derived at the objective level. ${ }^{7}$

At first glance, this result does not seem to rule out that an application of the Born rule at the objective level could be justified by its subjective implementation. In other words, one might hope that, though the Born rule can only be derived at the subjective level, its viability at this level justifies its use at the objective level as well. This possibility is blocked, however, by the fact that the decision theoretic derivation of the Born rule within the subjective perspective is based on disregarding parts of the objective spectrum of quantum states. The Born rule is only enforced on the individual agent once decoherence has provided the basis for ignoring macroscopically entangled states. The fact that macroscopic entangled states are suppressed thus clearly must be a consequence of the objective application of the Born rule but cannot possibly be a consequence of the decision theoretic argument where those states are not considered at all. The decision theoretic viability of the Born rule thus is a much less far-reaching claim than the objective viability of the Born rule, which makes it utterly impossible to infer the latter from the former.

Thus, we have that the proponent of the DWE scheme must implicitly accept a prohibition upon the objective implementation of the Born rule within their framework. When considered in combination with our reformulated version of the Zurek contention, one is then lead directly to a conceptual incoherence within the DWE argumentative structure:

- $\mathbf{Z}^{*}$ Decoherence cannot be practiced without an independent prior derivation (or assumption) of the Born rule as an objective probability measure.

- P1. Decoherence effects are a necessary precondition for one to assume the perspective of an agent making decisions (i.e. playing quantum games) within a world of distinct outcomes, corresponding to the discrete branches of the many worlds ontology.

- P2. Adopting this agential perspective is necessary prior to any Deutsch-Wallace type derivation of the Born rule as a subjective probability measure.

- P3. The viability of the DWE scheme rests upon there being no possible objective implementation of the Born rule in the Everettian framework.

Therefore:

- I1. The proponent of the DWE scheme must simultaneously assume: i) that the Born rule corresponds to an objective probability measure in order to appeal to the decoherence effects necessary to establish the Everettian ontology; and ii) that there is no possible objective probability measure available otherwise they cannot consistently establish their own subjective probability measure.

\footnotetext{
${ }^{7}$ Here again it should be noted that it might be more appropriate to frame our argument in terms of an ontological vs. epistemic distinction rather than objective vs. subjective. We retain the original terminology principally to avoid confusion.
} 
The interpretation of the wave function, necessary for decoherence, as associating an objective probability to states, thus is inconsistent with the core tenet of the subjectivist approach: the provision of a decision theoretical foundation for a purely subjective notion probability.

\subsection{Incoherence and ontological prejudice}

A further, incoherent, consequence of the chain of reasoning just given is that by appealing to an objective probabilistic notion of Born weights in order to justify neglecting superposition states, one would seem to have accepted such an interpretation of the Born weights of distinct states also. Thus we have that:

I2. In (effectively) eliminating superposition states due to their low Born weight the Everettian must either also (effectively) eliminate similarly low weighted distinct states and thus subvert their own position or simply apply a principle of ontological prejudice, such that superposition states are eliminated simply on the grounds of being superposition states, irrespective of their Born weighting.

Like other forms of prejudice, the essential problem is one of arbitrary discriminatory judgment: there is no basis for privileging one state over the other apart form belonging to a class that has been identified as privileged in principle. This second incoherence problem thus relates to the conceptual basis for justifying the many worlds ontology in the context of decoherence: On the one hand, in order to justify neglecting the superposition states the Everettian must assume that the Born weights represent objective probabilities of being realised - $\grave{a}$ la Copenhagen. On the other hand, in order to frame their many words branching ontology they must insist that all distinct states - even those with vanishingly small Born weights - are equally worthy of our attention. Prima facie, this seems completely inconsistent unless the Everettian can provide an alternative description of decoherence in which objective probabilities given by the Born rule are not assumed. Without such an alternative understanding of decoherence they would seem to either have to reject their own ontology (and thus give up their own interpretation!) or fall back to the endorsement of outright ontological prejudice: irrespective of Born weights the superposition states are neglected in favour of the distinct states merely on the virtue of the fact that they are superposition states. Thus, we have our point $\mathbf{I} 2$.

One possible line of response that could be made in defence of the DWE scheme is that the specifics of the decoherence effects are such that our second incoherence argument, relating to ontological prejudice, can be rebuffed. Our ontological prejudice charge is based upon the combination of the effective elimination of superposition states, with the retention of discrete states of comparably low Born weighting. Now, here the defender of the DWE approach might respond that, in practice, even the most unlikely discrete states will have a relatively much higher Born weighting than the highest weighted superposition states because of the extremely high level of suppression that decoherence effects enact. Whereas, the weightings of the off-diagonal elements fall off sharply with time, those associated with the least likely on-diagonal elements will only decrease according 
to the proliferation of branching due to measurement. Thus, there is no need for prejudice to establish as emergent a macro-regime that excludes superposition states but includes very unlikely discrete states.

Such a chain of reasoning is, however, not viable within the context of the neo-Everettian notions of branching and measurement. Under the DWE scheme, branching is not understood as being solely (or even principally) linked to deliberate human measurement events, rather it is understood as 'completely ubiquitous' [3, p.21] and to be mainly driven by random physical processes [17]. It is this notion of measurement that, when combined with decoherence, is intended to ground a discrete branching ontology within which the actual number of branches is fundamentally indeterminate. Given such a framework, the counter-argument to the prejudice charge outlined above fails: it is only by having an estimate of the number of branches on a given timescale (and thus also a concrete model of the branching process) that we can establish a quantification of the Born weighting associated with the lowest weighted discrete branch at that timescale, and without this there is no sense in which a comparison with the (suitably suppressed) superposition states can be made. And, of course, if one does admit a definite, enumerable branching structure, one immediately falls foul of the conflict between probabilities based upon branch counting, and those based upon the Born rule. Thus, even if one can construct an explicit model that justifies neglecting superposition states without prejudice, because it is predicated upon establishing a definite number of branches, the counter-argument would not merely fail to shield the DWE approach from the second incoherence charge, it would actually serve to entirely undermine the conceptual foundations upon which the scheme is built.

A simple example will further illustrate each of our two strong claims of incoherence. Let us again consider a simple two state system together with the detector as described above. Given that we have an appropriately repeatable preparation methodology, we can consider an arbitrarily large number of identical trials, $n$. For small $n$ decoherence involves us associating to states corresponding to sequences of distinct outcomes relatively high Born weights, and to the states corresponding sequences of non-district outcomes (i.e. superposition states) very low Born weights. By interpreting these Born weights as objective probabilities we could reasonably argue that the superposition states should be neglected. However if we do this and then want to apply an DWE type interpretation based on subjective probabilities, it seems we would have to forget about these objective probabilities as being relevant to the distinct states. This is the inconsistency problem I1. Let us then consider the situation for very large $n$, in that context we would have some states corresponding to sequences of distinct outcomes which have vanishingly small Born weights - e.g. all trials result in $|\uparrow\rangle$. Neglecting for the moment the inconsistency problem, if the Everettians apply an objective interpretation of the Born weights in order to eliminate the superposition states on the grounds of their very low probability then they must also eliminate, for large $n$, distinct 'all up' states on the grounds of their very low probability. But this the Everettian cannot do if they are to maintain their many world ontology. Thus, ontological prejudice as described above above becomes a necessity and we have $\mathbf{I} 2$. 


\section{$4 \quad$ Emergent worlds?}

The principal neo-Everettian bulwark against charges of conceptual incoherence, such as those presented above, must be expected to be some form of argument from emergence. In his [16], Wallace specifically responds to the weaker circularity version of our objection in such terms. In essence, the counter argument is that one may understand the Born weights as 'telling us when some emergent structure really is robustly present' [italics added]. This coincides with the treatment of [17], where a discrete branching structure is understood as a robust yet emergent feature of reality (p.15).

One may thus naturally anticipate a counter argument to our charges of incoherence that seeks to deflate the prior use of the Born measure by connecting it to the robustness of an emergent structure, rather than an objective probability rule. The notion is that one may say that the many worlds branching structure is robustly present in the quantum mechanical wavefunction in the same sense as tigers, phonons and galaxies are robustly present in the relevant underlying microphysics, but in making such a statement one need not apply the Born rule - i.e. interpret the Born measure in a probabilistic sense.

What we are to make of such claims regarding 'emergent structure' and 'robust feature of reality' is far from clear. On the one hand, it would be unreasonable to simply dismiss such language on grounds of vagueness. Although talk of emergence and structure is notoriously difficult to make precise, it has not gained considerable currency within both the philosophy of physics and the philosophy of science without good reason. ${ }^{8}$ Yet, on the other hand, we must be careful to make transparent and substantive characterisations of the claims involved otherwise their exact relevance to our incoherence charges will not be clear.

The first crucial distinction that can be made is between a notion of robustness that is empirically grounded and one that is not. By this we mean some qualification such that whether a structure within the formalism of a theory is taken to be robust is dependent upon some interpretational connection between that structure and empirical phenomenology. Clearly, the kind of thing that can provide empirical grounding to our notion of robustness is dependent upon the context at hand. The structure of a tigers, for example, might be established as a robust one via the consideration of a connection between an array of formal patters in our microphysics, and the zoological phenomena, such as, for example, eating philosophers! The micro-physical pattern corresponding to a coincidental arrangement of a tiger's tail resting in a glass of warm milk, on the other hand, cannot be understood as a robust structure in the same sense precisely because it fails to have any empirical grounding in zoology or otherwise. Clearly, in this case that the tiger-tail-milk structure fails to be empirically grounded can be taken to also imply that it has no explanatory value. In fact, in general, it seems reasonable to expect that any structure that has explanatory value within a scientific theory must also have some (possibly indirect) empirical grounding within that theory. However, the converse need not be true: we can think of structures that are empirically grounded

\footnotetext{
${ }^{8}$ The collection of readings in [22] gives a nice overview of the various scientific and philosophical perspectives on emergence that can be found in contemporary literature.
} 
but have no reasonable explanatory value - an uninterpreted set of observations for instance. Thus, our notion of the what it means for a structure to be robust is essentially weaker than the Dennett criterion invoked by Wallace in a similar context [16, p.50].

We can make the relevance of this grounding requirement to the case in hand immediately apparent by considering two possible structures within a quantum wavefunction. One we define via the reciprocal of the norm of the amplitudes components of states (relative to some basis). The other we define in the usual Born measure terms. Given an empirically grounded notion of robustness in terms of quantum mechanical measurements there is a clear sense in which the second structure could be understood as robust, but the first could not. Conversely, without empirical grounding via a link to phenomenology, designating a structure as 'robust' becomes a vacuous term and is unable to do the work required of it in differentiating between the two cases. Specifically, it would not help us understand why it is a reasonable idea to neglect very low Born weights based on the first structure, but a not so good one to neglect high Born weights based on the second.

Given this requirement that any 'robust structure' must be empirically grounded, we can then build towards a more precise characterisation of the 'emergent multiverse' claim such that the relevance (or not) for our incoherence problems can be accurately ascertained. To do this let us consider a exemplar of 'robust yet emergent structure' within physical theory taken from the framework of effective field theory (EFT) [23, 24, 25]. This is a useful case to consider since it: a) has an accompanying well developed scientific and philosophical literature from which a precise notion emergence can be drawn; and b) is reasonably analogous to the case of decoherence/many worlds with which we are concerned.

Following Bain [25], we can consider an EFT of a physical system as a theory of the dynamics of the system at energies small compared to a given cutoff (or characteristic energy threshold). In certain cases, the low energy states with respect to the cutoff are effectively independent of (decoupled from) states at high energies, and thus one may study the low-energy regime of the theory without the need for a detailed description of the high-energy regime. Many important physical systems admit a description in terms of EFTs (significant cases are found within particle physics, nuclear physics and condensed matter physics) and it is within the context of the ontological decoupling found within EFTs that we can consider a basic model for the concept of emergence that will be very useful to our analysis.

If we consider the pairing of an EFT and the relevant high-energy theory, then a precise notion of emergence is encapsulated within the the following (much simplified) framework: i) At energies low compared with the relevant cutoff a particular Lagrangian density provides a good description of the system; ii) At energies comparable or higher than the cutoff a different Lagrangian density provides a good description of the system; iii) The difference between these two mathematical formalisms is substantial enough to warrant them being interpreted in terms of different ontologies since they are defined based upon a different set field variables (and therefore different fundamental degrees of freedom); iv) In this context we can then define the ontology of the low energy effective theory as being emergent from that of the high-energy theory. 
Two crucial connections can be understood as necessary towards establishing this framework for defining emergence. The first connection is between the scaling of the relevant parameter (energy relative to the cutoff) and the empirical significance of particular aspects of the formalism (terms in the Lagrangian density): as the energy relative to the cutoff becomes low, the contribution of certain terms within the Lagrangian density become relatively much smaller than other terms. The second connection is between the significance of these formal structures (smallness of the terms) and their eliminablity from the formalism (Lagrangian density): the terms which are relatively much smaller are removed from the Lagrangian density because they are less empirically significant. ${ }^{9}$ Whereas, the first connection is established purely by the form of the relevant equations, the second relies on our interpretation of small parameter values of terms within the Lagrangian density as implying the negligible physical relevance of whatever ontological structures can be associated with those terms. Given this interpretational step leading to the second condition, we can then give a precise meaning to the statement that 'the ontology of the EFT is emergent'; but without it there is a gap in our chain of reasoning.

We thus again run into the subtle, yet crucial, issue of needing a physical basis for neglecting small parameter values: we have to establish that the magnitude of a term within the Lagrangian density is related to its effect on the measurement to be performed. Now, for the case of EFTs we of course have a fairly straightforward basis for making the necessary connection between the formal and the empirical. Terms which make a very small contribution to the Lagrangian density will make a correspondingly small contribution to the relevant S-matrix. Given an interpretation of this matrix in probabilistic terms (via its connection to the relevant scattering amplitudes), we can then establish that neglecting very small terms in the Lagrangian density will have a negligible effect to any measurement performed on the system. Thus, we have a solid basis for the relevant interpretational step, and can consider the ontology of the low level theory as a 'robust but emergent structure' in a sense that has the necessary empirically grounding.

Clearly, there is much more that could be said on these issue, all we have here is a rather simplistic scheme for characterising both emergence and EFTs. However, given this analysis we can at least provide a substantive idea of what it means to say that 'the ontology of the EFT is emergent'. It would seem reasonable to require the notion of emergence deployed in the defence of an Everettian ontology to be as least as substantive as this basic sketch.

Let us then distill the key elements of this notion of emergence, as we have defined it in the context of effective field theory, into a a more general schema applicable to the many worlds/decoherence case. In essence what we had in the EFT case was: i) a clear notion of the the parameter that is being scaled between the two regimes; ii) two distinct mathematical formalisms, one relevant to each regime with the formal distinction established (at least partially) via the scaling of the parameter; and iii) an explicit basis for connecting the difference between the two formalisms to

\footnotetext{
${ }^{9}$ This crucial feature can be seen explicitly within the 'Wilsonian EFT' scheme where the irrelevant terms are neglected on precisely the basis of scaling in powers of $E / \Lambda$ (where $E$ is the energy scale and $\Lambda$ is the cutoff). It is also implicit within the 'continuum EFT' scheme since a similar discarding of terms due to scaling behaviour is needed to calculate the matching correction. See [25].
} 
an ontological distinction. Together these three aspects were taken as sufficient to establish an emergent ontology in the EFT case - we should therefore accept something similar as establishing a discrete branching structure as an emergent ontology.

Environment induced decoherence is a process with effects that scale with both time and complexity: on longer timescales and in more complex systems decoherence effects become greater. It is natural, then, to think of the two regimes that we are considering as being: a short time scale, low complexity regime; and a longer time scale high complexity regime. For simplicity we shall simply call them the 'macro-regime' and 'micro-regime', and consider a basic model of decoherence (along the lines of [18, pp.12-14]) within which the time parameter controls the suppression of the relevant off-diagonal terms. We thus have that in the micro-regime the relevant mathematical formalism is a density matrix of the form $\rho^{C}$ above, and in the macro-regime the relevant mathematical formalism is a density matrix of the form $\rho^{R}$. There is an explicit basis for considering this formal difference as grounding an ontological difference since the states that can be represented through the formalism relevant to the micro-regime include the discrete and superposition states, and those that can be represented by the formalism relevant to the micro-regime include only discrete states.

We are therefore in a position to call the ontology of the macro-regime a robustly emergent structure, provided the two crucial connections discussed above hold. Firstly, we need to connect the scaling of the relevant parameter (time) and the significance of particular aspects of the formalism (off-diagonal elements of the density matrix). In Zurek's simple model [18, pp.12-14] this connection obtains explicitly since the 'decoherence term' in his Eq. 17 leads to a strong decay (i.e. reduction with respect to time) in the off-diagonal elements, but has negligible effect on the on-diagonal elements. Thus, the first connection holds. The second connection required is between the relative smallness of these off-diagonal density matrix elements and their eliminablity from the formalism. Above we saw that in order for this connection to hold we need a physical basis for the interpretational step of neglecting small values. In order to justify neglecting very small entries within a density matrix we need to connect this formal structure to some empirical structure: we must argue that neglecting low Born weighted states has a negligible impact upon measurements of the system. Thus we must apply some version of the Born rule as an objective measure of probability of experimental outcomes. We therefore, have that without the objective Born rule we cannot coherently claim that the ontology of the macro-regime emerges, and so arrive at precisely the logical structure with regard to subjective/objective probability, decoherence, and many worlds quantum mechanics as was detailed above. Our two incoherence arguments may thus again be brought to bare, and the position for the proponent of a DWE type scheme becomes just as fraught as before.

\section{Conclusion}

The Deutsch-Wallace-Everett programme can be understood as conceptually incoherent since its viability rests upon a notion of decoherence that conflicts with its own fundamental precepts with 
regard to both the subjective nature of probability, and the discrete nature of ontology. Under careful analysis, these problems are not alleviated by invoking the notion of emergent or robust structure, and thus, as it stands, our twin issues of incoherence must be seen as rendering the scheme deeply inadequate as an approach to quantum theory.

Some ideas are too attractive to be considered false; and some are too ugly to be considered true. Still others, despite their apparent beauty, turn out simply inconsistent, no matter how seductive they may be at first sight. This third category of elegant incoherence carries the highest risk of leading astray, and it is there, alas, that the seemingly revolutionary Deutsch-Wallace approach to Everettian quantum theory must be situated.

\section{References}

[1] D. Deutsch, "Quantum theory of probability and decisions," Proceedings of the Royal Society of London. Series A: Mathematical, Physical and Engineering Sciences 455 no. 1988, (1999) 3129-3137, http://rspa.royalsocietypublishing.org/content/455/1988/3129.full.pdf+html. http://rspa.royalsocietypublishing.org/content/455/1988/3129. abstract.

[2] D. Wallace, "Quantum probability and decision theory, revisited," quant-ph/0211104v1. http://arxiv.org/abs/quant-ph/0211104v1.

[3] D. Wallace, "Quantum Probability from Subjective Likelihood: improving on Deutsch's proof of the probability rule," Studies In History and Philosophy of Science Part B: Studies In History and Philosophy of Modern Physics 38 no. 2, (2007) 311-332.

[4] D. Wallace, "A formal proof of the born rule from decision-theoretic assumptions," ArXiv e-prints http://arxiv.org/abs/0906.2718v1 (2009), 0906.2718v1. http://arxiv.org/abs/0906.2718v1.

[5] S. Saunders, "Derivation of the born rule from operational assumptions," Proceedings of the Royal Society of London. Series A: Mathematical, Physical and Engineering Sciences 460 no. 2046, (2004) 1771-1788, http://rspa.royalsocietypublishing.org/content/460/2046/1771.full.pdf+html. http://rspa.royalsocietypublishing.org/content/460/2046/1771. abstract.

[6] H. Price, "Decisions, Decisions, Decisions: Can Savage Salvage Everettian Probability?," in Many Worlds? Everett, Quantum Theory, and Reality, S. Saunders, J. Barrett, A. Kent, and D. Wallace, eds., pp. 369-391. Oxford Univeristy Press, 2010.

[7] P. Lewis, "Uncertainty and probability for branching selves," Studies In History and Philosophy of Science Part B: Studies In History and Philosophy of Modern Physics 38 no. $1,(2006) 1 / 14$. 
[8] M. Hemmo and I. Pitowsky, "Quantum probability and many worlds," Studies In History and Philosophy of Science Part B: Studies In History and Philosophy of Modern Physics $\mathbf{3 8}$ (2007) 333-350.

[9] W. H. Zurek, "Probabilities from entanglement, Born's rule $p_{k}=\left|\psi_{k}\right|^{2}$ from envariance," Phys. Rev. A 71 (May, 2005) 052105. http://link.aps.org/doi/10.1103/PhysRevA.71.052105.

[10] W. H. Zurek, "Quantum jumps, Born's rule, and objective reality," in Many Worlds? Everett, Quantum Theory, and Reality, S. Saunders, J. Barrett, A. Kent, and D. Wallace, eds., ch. 13, pp. 409-432. Oxford Univeristy Press, 2010.

[11] D. J. Baker, "Measurement outcomes and probability in everettian quantum mechanics," Studies In History and Philosophy of Science Part B: Studies In History and Philosophy of Modern Physics 38 no. 1, (2007) 153 - 169. http://www.sciencedirect.com/science/article/pii/S1355219806000694.

[12] A. Kent, "One world versus many: The inadequacy of Everettian accounts of evolution, probability, and scientific confirmation," in Many Worlds? Everett, Quantum Theory, and Reality, S. Saunders, J. Barrett, D. Wallace, and A. Kent, eds., ch. 10, pp. 307-355. Oxford Univeristy Press, 2010. http:

//www. ingentaconnect. com/content/oso/6510144/2010/00000001/00000001/art00016.

[13] D. Albert, "Probablity in the everett picture," in Many Worlds? Everett, Quantum Theory, and Reality, S. Saunders, J. Barrett, D. Wallace, and A. Kent, eds., ch. 11, pp. 354-368. Oxford Univeristy Press, 2010. http: //www. ingentaconnect. com/content/oso/6510144/2010/00000001/00000001/art00016.

[14] A. I. Rae, "Everett and the Born rule," Studies In History and Philosophy of Science Part B: Studies In History and Philosophy of Modern Physics 40 no. 3, (2009) 243 - 250. http://www.sciencedirect.com/science/article/pii/S1355219809000306.

[15] S. Saunders, "Time, quantum mechanics, and probability," Synthese 114 no. 3, (1998) 373-404.

[16] D. Wallace, The Emergent Multiverse. Oxford Univeristy Press, 2012.

[17] D. Wallace, "Decoherence and ontology," in Many Worlds? Everett, Quantum Theory, and Reality, S. Saunders, J. Barrett, A. Kent, and D. Wallace, eds., ch. 1, pp. 34-72. Oxford Univeristy Press, 2010.

[18] W. H. Zurek, "Decoherence and the transition from quantum to classical - revisited," Physics Today 44 no. 10, (2003) 2-37, quant-ph/0306072v1. http://arxiv.org/abs/quant-ph/0306072v1. 
[19] A. Valentini and H. Westman, "Dynamical origin of quantum probabilities," Royal Society of London Proceedings Series A 461 (Jan., 2005) 253-272, arXiv:quant-ph/0403034.

[20] S. Saunders, "What is probability?," in Quo Vadis Quantum Mechanics?, E. Avshalom, S. Dolev, and N. Kolenda, eds., The Frontiers Collection, pp. 209-238. Springer, 2005.

[21] D. Lewis, "A subjectivist's guide to objective chance," in Studies in Inductive Logic and Probability Vol. II, R. C. Jeffrey, ed., pp. pp. 263-93. University of California Press, 1980.

[22] B. M. A. and P. Humphreys, eds., Emergence: Contemporary Readings in Philosophy and Science. A Bradford Book, 2008.

[23] H. Georgi, "Effective field theory," Annual Review of Nuclear and Particle Science 43 no. 1, (1993) 209-252.

[24] S. Hartmann, "Effective field theories, reductionism and scientific explanation," Studies In History and Philosophy of Science Part B: Studies In History and Philosophy of Modern Physics 32 no. 2, (2001) 267-304.

[25] J. Bain, "Effective field theories." http://Is.poly.edu/ jbain/papers/EFTs.pdf. 